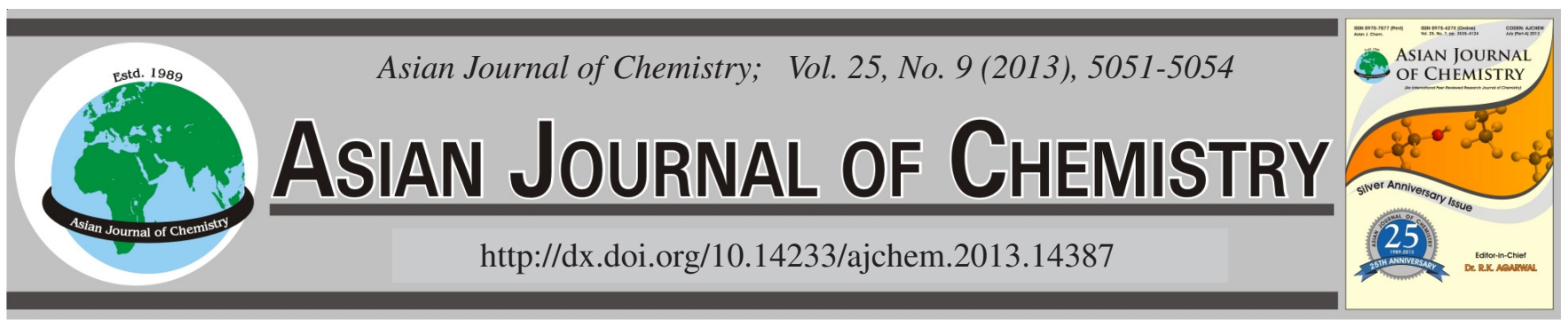

\title{
Analysis of Phytochemical Constituent of the Volatile Oil in the Wedelia biflora by HS-SPME/GC-MS
}

\author{
Chao-Feng Yang, Yu-Qiu Huang and Ke Yuan*
}

The Nurturing Station for the State Key Laboratory of Subtropical Silviculture, Zhejiang Agriculture and Forestry University, Lin'an 311300 , P.R. China

*Corresponding author: Tel: +86 571 63743607; E-mail: yuan_ke001@163.com

(Received: 2 August 2012;

Accepted: 20 March 2013)

AJC-13135

\begin{abstract}
To analyze the chemical constituents of the volatile oil in the green peel of Wedelia biflora, the chemical constituents was extracted from the Wedelia biflora by solid-phase microextraction. The chemical constituents of the volatile oil separated and identified by GC-MS. The relative content of each component was determined by area normalization. Seventy-two kinds of components were separated. Among them, 68 components were identified, accounting $c a$. $98.51 \%$ of the total chemical constituents, most of them belonged to sesquiterpenoids, the main chemical constituents of the Wedelia biflora are D-limonene (15.40\%); 4,11,11-trimethyl-8-methylene-[1R-(1R*,4Z,9S*)]bicyclo[7.2.0]undec-4-ene (9.70\%); 1R- $\alpha$-Pinene (8.94\%); 4-methyl-1-(1-methylethyl)-didehydro derive bicyclo[3.1.0]hexane (8.07 \%); 1-methyl-3- (1-methylethyl)-benzene (6.71\%); 1,1,4,8-tetramethyl- cis, cis, cis-4,7,10-cycloundecatriene (5.39\%); germacrene D (4.65\%), etc. The result of the experiments can provide reference for further research on the chemical components of Wedelia biflora volatile oil as well as for the exploration and utilization of it.
\end{abstract}

Key Words: Wedelia biflora, Volatile oil, Solid-phase microextraction, GC-MS.

\section{INTRODUCTION}

Wedelia biflora (Linn.) DC., also called chrysanthemum sesarmid crab, chrysanthemum ink vegetables, chrysanthemum dragon tongue plant, Tianhuang Ju and Ludiju, etc. It is used as medicine entirely ${ }^{1-3}$. We usually harvest this herb in summer and fall and then wash it clean, keep it fresh and sun-dried. The Wedelia biflora (Linn.) DC. is distributed in the Oceania, Indo-china peninsula, the Philippines, India, Japan, Taiwan Island, Malaysia, Indonesia and in the regions of the Chinese Mainland, such as Guangdong, Hainan, Guangxi and Yunnan, etc. According to the literature, Wedelia biflora (Linn.) DC. has the pharmacologic functions ${ }^{4-6}$ of liver-protection, antivirus, antitumors, antiinflammation and analgesia. In order to make full use of this resource, this work conducts a study on the chemical compositions of its volatile oil.

Solid-phase microextraction (SPME) ${ }^{7-10}$ is a new technology put forward by the scientists like Pawliszyn in 1990. This method doesn't use organic solvent. It is easy and convenient, combining the following processes together: herbs-colleting, extracting, concentration and sample-feeding. And thus it has the advantages of fast analysis (generally it can reach absorbing balance within 2-30 min), high sensitivity and good repeatability. In view of its above advantages, this research uses the method of solid-phase microextraction for the first time to extract the chemical compositions in Wedelia biflora (Linn.) DC produced in Hainan Island. It adopts the coupling technique of gas chromatograph-mass spectrometer to analyze and determine its chemical compositions. Overall, it separated 72 components and determined 68 compounds. The determined components takes up $98.51 \%$ of the total peak area. The relative contents of each peak can be calculated by the peakarea normalization method, which provides a reference for the further research of its active components in the volatile oil and its further exploitation of this natural resource.

\section{EXPERIMENTAL}

Trace MS Gas Chromatograph-tandem Mass Spectrometer (American Phenegon Company); Chromatographic Column; DB-WAX (30 m × $0.25 \mathrm{~mm}, 0.25 \mathrm{~mm})$ Quarts Capillary Column; Manual Solid-phase Microextraction, SPME (American Supelco Company); the extracting fiberhead is $65 \mu \mathrm{m}$ PDMS/DVB; the mixed alkane standard samples of $\mathrm{C}_{8}-\mathrm{C}_{20}$ and $\mathrm{C}_{21}-\mathrm{C}_{40}$.

The Wedelia biflora (Linn.) DC. was picked in Sanya, Hainan Island in August, 2011. It was dried and kept in the dry containers for future use after being cleansed, crushed and sieved from the 100-mesh sift. 
Solid-phase microextraction conditions: Weight $1.5 \mathrm{~g}$ powder of the Wedelia biflora (Linn.) DC and put it in the special sample-taking bottle of $15 \mathrm{~mL}$ and then insert it into the manual sample-feeding machine with the fiber-head of 65 $\mu \mathrm{m}$ PDMS/DVB. Its magnetic stirring speed is $1100 \mathrm{rpm}$; take away after $0.5 \mathrm{~h}$ of extraction at $90^{\circ} \mathrm{C}$ and immediately insert it into the sample-feeding mouth of the chromatograph (the temperature is $250{ }^{\circ} \mathrm{C}$ ) with 2.5 min of desorption.

Analytical condition of the gas chromatograph-mass spectrometer: The gas chromatograph condition: the chromatographic column is the quarts capillary column of DBWAX $(30 \mathrm{~m} \times 0.25 \mathrm{~mm}, 0.25 \mathrm{~mm})$; temperature-raising process:the initial temperature is $45^{\circ} \mathrm{C}$ for $3 \mathrm{~min}$ and then raise the temperature to $100{ }^{\circ} \mathrm{C}$ at the speed of $10^{\circ} \mathrm{C} \mathrm{min}^{-1}$ and keep it for $15 \mathrm{~min}$, then again raise it to $170^{\circ} \mathrm{C}$ at the speed of $5^{\circ} \mathrm{C} \mathrm{min}{ }^{-1}$; raise it to $240{ }^{\circ} \mathrm{C}$ for $7 \mathrm{~min}$ at the speed of $10{ }^{\circ} \mathrm{C}$ $\mathrm{min}^{-1}$; the temperature at the sample-feeding mouth is $250^{\circ} \mathrm{C}$, the temperature at the evaporating room is $250{ }^{\circ} \mathrm{C}$; the carrier gas is helium (He) and its flow velocity is $0.8 \mathrm{~mL} \mathrm{~min}^{-1}$ and there is no bypass flow with its sample amount of $1.0 \mathrm{~mL}$ and its split ratio is $50: 1$.

The mass-spectrum condition:electron bombardment ion source (EI); ionizing energy is $70 \mathrm{eV}$, the temperature of the ion source is $200{ }^{\circ} \mathrm{C}$, the voltage of the detector is $350 \mathrm{~V}$, the detection range is between $40-300 \mathrm{~m} \mathrm{z}^{-1}$ and the detecting interval is $0.5 \mathrm{~s}$. The scanning mass range is $\mathrm{m} / \mathrm{z}: 333-500$ AMU; the scanning speed is $0.5 \mathrm{~s}$; the retrieval atlas databanks are the standard mass spectrum map depot of Willey and NIST.

Calculation of retention indices: This quasi-linear equation proposed by Van den Dool and $\mathrm{Kratz}^{6}$ was used to calculate retention indices in this work:

$$
\mathrm{IT}=100 \times \mathrm{n}+100 \times \frac{(\mathrm{tx}-\mathrm{tn})}{(\mathrm{tn}+1-\mathrm{tn})}
$$

where IT is the temperature-programmed retention index of the interesting compound and $\mathrm{tn}, \mathrm{tn}+1$ and $\mathrm{tx}$ are the retention times of the two standard $n$-alkanes containing $n$ and $n+1$ carbons and the compound of interest, respectively.

Determining results: Adopt the DB-WAX capillary column, take the suitable amount of the sample, analyze and determine by gas chromatograph-mass spectrometer and we get the total volatile oil ion flow chart of the Wedelia biflora (Linn.) DC. (Fig. 1). After the mass-spectrum scanning for each chromatographic peak, we get the mass-spectrum graph. We also consult the relevant mass-spectrum data through the mass-spectrum bank retrieval of NIST2008 standard. The compound quantity takes the area normalization method and we calculate each peak area by the Hewlett-Packard software system for the purpose of getting the relative percentage in each volatile oil component. Then calculate their RI values by choosing the possible matter with high matching degree with the mass-spectrum. And finally we determined the chemical composition of the volatile oil in the Wedelia biflora (Linn.) DC. by combining the artificial analysis (Table-1).

Fig. 1 showed that there are 68 kinds of chemical components in the volatile oil of the Wedelia biflora (Linn.) DC. The main contents are diterpene and aromatic components of micromolecules. Among them the high-content components are: Component 1: D-Limonene (15.40\%); component 2:

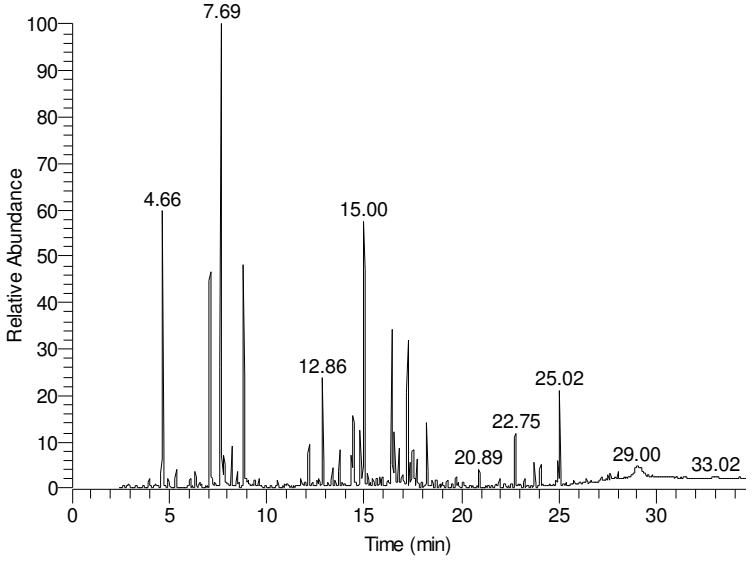

Fig. 1. GC-MS total ion current chromatogram of the volatile oil in the Wedelia biflora

4,11,11-Trimethyl-8-methylene-[1R-(1R*,4Z,9S*)]bicyclo[7.2.0]undec-4-ene $(9.70 \%)$; component 3: $1 \mathrm{R}-\alpha-$ Pinene (8.94\%); component 4: 4-Methyl-1-(1-methylethyl)didehydro derive bicyclo[3.1.0]hexane (8.07\%); component 5: 1-methyl-3-(1-methylethyl)-benzene (6.71\%); component 6: 1,1,4,8-tetramethyl-cis, cis, cis-4,7,10-cycloundecatriene (5.39\%); component 7: Germacrene D (4.65\%); component 8: copaene (2.90\%); component 9: Octahydro-7-methyl-3methylene-4-(1-methylethyl)- [3 $\left.\alpha \mathrm{S}-\left(3 \alpha \alpha, 3 \alpha \beta, 4 \alpha, 7 \alpha, 7 \alpha \mathrm{S}^{*}\right)\right]-$ $1 H$-cyclopenta[1,3]cyclopropa[1,2]benzene $(2.46 \%)$; component 10: $1,2,4 \alpha, 5,8,8 \alpha$-hexahydro-4,7-dimethyl-1-(1methylethyl)-[1S-( $1 \alpha, 4 \alpha \alpha, 8 \alpha \alpha)]$-naphthalene (2.20\%); component 11: 2,3,4,7,8,8 $\alpha$-hexahydro-3,6,8,8-tetramethyl-[3R( $3 \alpha, 3 \alpha \alpha, 7 \alpha, 8 \alpha \alpha)]-1 H-3 \alpha, 7$-methanoazulene (2.10\%); component 12: Octahydro-3,8,8-trimethyl-6-methylene-, [3R$(3 \alpha, 3 \alpha \alpha, 7 \alpha, 8 \alpha \alpha)]-1 H-3 \alpha, 7$-methanoazulene (1.96\%); component 13: caryophyllene oxide $(1.53 \%)$, structure of components are given in Fig. 2.

\section{RESULTS AND DISCUSSION}

It can also be seen in Table- 1 that we can get 68 kinds of components by using solid-phase microextraction to extract the chemical compositions of the volatile oil in the Wedelia biflora (Linn.) DC. and by using gas chromatograph-tandem mass spectrometer to analyze and determine. The determined components take up $98.51 \%$ of the total peak area. Its main components are: D-Limonene (15.40\%); 4,11,11-trimethyl8-methylene-[1R-(1R*,4Z,9S*)]-bicyclo[7.2.0] undec-4-ene (9.70\%); 1R- $\alpha$-pinene (8.94\%); 4-methyl-1- (1-methylethyl)didehydro derive bicyclo[3.1.0]hexane (8.07\%); 1-methyl-3(1-methylethyl)-benzene $(6.71 \%) ; 1,1,4,8$-tetramethylcis,cis,cis-4,7,10-cycloundecatriene (5.39\%); germacrene D (4.65\%); copaene $(2.90 \%)$; octahydro-7-methyl-3-methylene-4-(1-methylethyl)-[3 $\left.\alpha \mathrm{S}-\left(3 \alpha \alpha, 3 \alpha \beta, 4 \alpha, 7 \alpha, 7 \alpha \mathrm{S}^{*}\right)\right]-1 H$ cyclopenta[1,3]cyclopropa[1,2] benzene $(2.46 \%)$; $1,2,4 \alpha, 5,8,8 \alpha$-hexahydro-4,7-dimethyl-1-(1-methylethyl)[1S- $(1 \alpha, 4 \alpha \alpha, 8 \alpha \alpha)]$-naphthalene $(2.20 \%) ; 2,3,4,7,8,8 \alpha-$ hexahydro-3,6,8,8-tetramethyl-[3R-(3 $\alpha, 3 \alpha \alpha, 7 \alpha, 8 \alpha \alpha)]-1 H$ $3 \alpha, 7$-methanoazulene $(2.10 \%)$; octahydro-3,8,8-trimethyl-6-

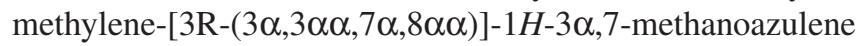
$(1.96 \%)$; caryophyllene oxide $(1.53 \%)$. 
TABLE-1

ANALYTICAL RESULTS OF CHEMICAL CONSTITUENTS OF THE VOLATILE OIL IN THE Wedelia biflora

\begin{tabular}{|c|c|c|c|c|c|}
\hline No. & Name of components & RT (min) & m.f. & RI & Relative concent $(\%)$ \\
\hline 1 & 3-Pentanone & 3.98 & $\mathrm{C}_{5} \mathrm{H}_{10} \mathrm{O}$ & 979.433 & 0.37 \\
\hline 2 & 1R- $\alpha$-Pinene & 4.66 & $\mathrm{C}_{10} \mathrm{H}_{16}$ & 1022.543 & 8.94 \\
\hline 3 & Toluene & 4.96 & $\mathrm{C}_{7} \mathrm{H}_{8}$ & 1039.884 & 0.29 \\
\hline 4 & Camphene & 5.35 & $\mathrm{C}_{10} \mathrm{H}_{16}$ & 1062.428 & 0.39 \\
\hline 5 & Bicyclo[3.1.1]heptane, 6,6-dimethyl-2-methylene-, (1S)- & 6.09 & $\mathrm{C}_{10} \mathrm{H}_{16}$ & 1105.263 & 0.29 \\
\hline 6 & $\alpha$-Phellandrene & 6.34 & $\mathrm{C}_{10} \mathrm{H}_{16}$ & 1119.883 & 0.42 \\
\hline 7 & 3-Hexen-2-one & 6.56 & $\mathrm{C}_{6} \mathrm{H}_{10} \mathrm{O}$ & 1132.749 & 0.17 \\
\hline 8 & 4-Methyl-1-(1-methylethyl)-didehydro derive bicyclo[3.1.0]hexane & 7.10 & $\mathrm{C}_{10} \mathrm{H}_{16}$ & 1164.327 & 8.07 \\
\hline 9 & 1-Methyl-4-(1-methylethyl)-1,4-cyclohexadiene & 7.34 & $\mathrm{C}_{10} \mathrm{H}_{16}$ & 1178.363 & 0.23 \\
\hline 10 & D-Limonene & 7.69 & $\mathrm{C}_{10} \mathrm{H}_{16}$ & 1198.830 & 15.40 \\
\hline 11 & 4-Methyl-1-(1-methylethyl)-bicyclo[3.1.0]hex-2-ene & 7.82 & $\mathrm{C}_{10} \mathrm{H}_{16}$ & 1206.707 & 0.96 \\
\hline 12 & 3,6,6-Trimethyl-bicyclo[3.1.1]hept-2-ene & 8.23 & $\mathrm{C}_{10} \mathrm{H}_{16}$ & 1231.707 & 0.83 \\
\hline 13 & 2-Methyl-5-(1-methylethyl)-(1 $\alpha, 2 \alpha, 5 \alpha)$-bicyclo[3.1.0]hexan-2-ol & 8.41 & $\mathrm{C}_{10} \mathrm{H}_{21} 0_{18}$ & 1242.683 & 0.14 \\
\hline 14 & 3,7-Dimethyl-(Z)-1,3,6-octatriene, & 8.50 & $\mathrm{C}_{10} \mathrm{H}_{16}$ & 1248.171 & 0.35 \\
\hline 15 & 3-Octanone & 8.56 & $\mathrm{C}_{8} \mathrm{H}_{16}$ & 1251.829 & 0.15 \\
\hline 16 & 1-Methyl-3-(1-methylethyl)-benzene & 8.83 & $\mathrm{C}_{10} \mathrm{H}_{14}$ & 1268.293 & 6.71 \\
\hline 17 & $1,3,8-p$-Menthatriene & 9.03 & $\mathrm{C}_{10} \mathrm{H}_{14}$ & 1280.488 & 0.30 \\
\hline 18 & 2-Ethenyl-1,1-dimethyl-3-methylene-cyclohexane & 9.40 & $\mathrm{C}_{11} \mathrm{H}_{18}$ & 1302.793 & 0.14 \\
\hline 19 & 3,6-Dimethoxy-9-(2-phenylethynyl)-fluoren-9-ol & 9.61 & $\mathrm{C}_{23} \mathrm{H}_{18} \mathrm{O}_{3}$ & 1314.525 & 0.16 \\
\hline 20 & 4,4,6,6-Tetramethyl-bicyclo[3.1.0]hex-2-ene & 10.53 & $\mathrm{C}_{10} \mathrm{H}_{16}$ & 1365.922 & 0.15 \\
\hline 21 & 1-methyl-4-(1-methylethenyl)-benzene & 11.74 & $\mathrm{C}_{10} \mathrm{H}_{12}$ & 1430.928 & 0.34 \\
\hline 22 & 1-Octen-3-ol & 11.97 & $\mathrm{C}_{8} \mathrm{H}_{16} \mathrm{O}$ & 1442.784 & 0.16 \\
\hline 23 & $\alpha$-Cubebene & 12.17 & $\mathrm{C}_{15} \mathrm{H}_{24}$ & 1453.093 & 1.05 \\
\hline 24 & $\begin{array}{l}\text { 1-Ethenyl-1-methyl-2-(1-methylethenyl)-4-(1-methylethylidene)- } \\
\text { cyclohexane }\end{array}$ & 12.60 & $\mathrm{C}_{15} \mathrm{H}_{24}$ & 1475.258 & 0.13 \\
\hline 25 & Ylangene & 12.67 & $\mathrm{C}_{15} \mathrm{H}_{24}$ & 1478.866 & 0.27 \\
\hline 26 & Copaene & 12.86 & $\mathrm{C}_{15} \mathrm{H}_{24}$ & 1488.660 & 2.90 \\
\hline 27 & $\begin{array}{l}\text { Decahydro-3 } \alpha \text {-methyl-6-methylene-1-(1-methylethyl)-[1S- }(1 \alpha, 3 \alpha \alpha, 3 \alpha \beta, \\
6 \alpha \alpha, 6 \alpha \beta)] \text {-cyclobuta[1,2:3,4]dicyclopentene }\end{array}$ & 13.38 & $\mathrm{C}_{15} \mathrm{H}_{24}$ & 1514.778 & 0.52 \\
\hline 28 & Di-epi-à-cedrene-(I) & 13.49 & $\mathrm{C}_{15} \mathrm{H}_{24}$ & 1520.197 & 0.16 \\
\hline 29 & $\begin{array}{l}\text { Octahydro-7-methyl-3-methylene-4-(1-methylethyl)-[3 } \alpha \mathrm{S}-(3 \alpha \beta, 3 \alpha \beta, 4 \alpha, 7 \alpha \text {, } \\
\left.\left.7 \alpha \mathrm{S}^{*}\right)\right]-1 H \text {-cyclopenta[1,3]cyclopropa[1,2]benzene }\end{array}$ & 13.74 & $\mathrm{C}_{15} \mathrm{H}_{24}$ & 1532.512 & 2.46 \\
\hline 30 & $\begin{array}{l}\text { 2,3,4,7,8,8 } \alpha \text {-Hexahydro-3,6,8,8-tetramethyl-[3R- }(3 \alpha, 3 \alpha \alpha, 7 \alpha, 8 \alpha \alpha)]-1 H-3 \alpha, \\
\text { 7-methanoazulene }\end{array}$ & 14.39 & $\mathrm{C}_{15} \mathrm{H}_{24}$ & 1564.532 & 2.10 \\
\hline 31 & 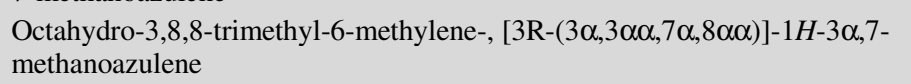 & 14.47 & $\mathrm{C}_{15} \mathrm{H}_{24}$ & 1568.473 & 1.96 \\
\hline 32 & 1-Ethenyl-1-methyl-2,4-bis(1-methylethenyl)-[1S-(1 $\alpha, 2 \alpha, 4 \alpha)]$-cyclohexane & 14.79 & $\mathrm{C}_{15} \mathrm{H}_{24}$ & 1584.236 & 1.60 \\
\hline 33 & $\begin{array}{l}\text { 4,11,11-Trimethyl-8-methylene-[1R-(1R*,4Z,9S*)]-bicyclo[7.2.0]undec-4- } \\
\text { ene }\end{array}$ & 15.00 & $\mathrm{C}_{15} \mathrm{H}_{24}$ & 1594.581 & 9.70 \\
\hline 34 & 2-Methyl-5-(1-methylethenyl)-cyclohexanone & 15.17 & $\mathrm{C}_{10} \mathrm{H}_{16} \mathrm{O}$ & 1602.871 & 0.33 \\
\hline 35 & Thujopsene & 15.46 & $\mathrm{C}_{15} \mathrm{H}_{24}$ & 1616.746 & 0.20 \\
\hline 36 & $\alpha$-Cubebene & 15.65 & $\mathrm{C}_{15} \mathrm{H}_{24}$ & 1625.837 & 0.24 \\
\hline 37 & $\begin{array}{l}\text { Decahydro-1,1,7-trimethyl-4-methylene-[1 } \alpha \mathrm{R}-(1 \alpha \alpha, 4 \alpha \alpha, 7 \alpha, 7 \alpha \alpha, 7 \alpha \beta)] \text { - } \\
1 H \text {-cycloprop[e]azulene }\end{array}$ & 15.94 & $\mathrm{C}_{15} \mathrm{H}_{24}$ & 1639.713 & 0.29 \\
\hline 38 & 1,1,4,8-Tetramethyl-cis, cis, cis-4,7,10-cycloundecatriene & 16.44 & $\mathrm{C}_{15} \mathrm{H}_{24}$ & 1663.636 & 5.39 \\
\hline 39 & 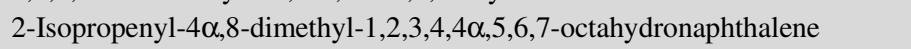 & 16.54 & $\mathrm{C}_{15} \mathrm{H}_{24}$ & 1668.421 & 1.65 \\
\hline 40 & 4,6,6-Trimethyl-[1S-(1 $\alpha, 2 \alpha, 5 \alpha)]$-bicyclo[3.1.1]hept-3-en-2-ol & 16.65 & $\mathrm{C}_{10} \mathrm{H}_{16} \mathrm{O}$ & 1673.684 & 0.29 \\
\hline 41 & (+)-Epi-bicyclosesquiphellandrene & 16.73 & $\mathrm{C}_{15} \mathrm{H}_{26}$ & 1677.512 & 0.21 \\
\hline 42 & $\begin{array}{l}1,2,3,4,4 \alpha, 5,6,8 \alpha \text {-Octahydro-7-methyl-4-methylene-1-(1-methylethyl)-( } 1 \alpha \\
4 \alpha \alpha, 8 \alpha \alpha) \text {-naphthalene }\end{array}$ & 16.80 & $\mathrm{C}_{15} \mathrm{H}_{24}$ & 1680.861 & 1.34 \\
\hline 43 & $\alpha, \alpha, 4$-Trimethyl-, acetate 3-cyclohexene-1-methanol & 16.94 & $\mathrm{C}_{12} \mathrm{H}_{20} \mathrm{O}_{2}$ & 1687.56 & 0.21 \\
\hline 44 & $\alpha, \alpha, 4$-Trimethyl-, (S)-3-cyclohexene-1-methanol & 17.00 & $\mathrm{C}_{10} \mathrm{H}_{18} \mathrm{O}$ & 1690.431 & 0.27 \\
\hline 45 & Germacrene D & 17.24 & $\mathrm{C}_{15} \mathrm{H}_{24}$ & 1701.923 & 4.65 \\
\hline 46 & $\begin{array}{l}\text { Decahydro- } 4 \alpha-\text {-methyl-1-methylene-7-(1-methylethenyl)-[4 } \alpha \mathrm{R}-(4 \alpha \alpha, 7 \alpha, \\
8 \alpha \alpha)] \text {-naphthalene }\end{array}$ & 17.45 & $\mathrm{C}_{15} \mathrm{H}_{24}$ & 1712.019 & 1.13 \\
\hline 47 & $\alpha$-Muurolene & 17.52 & $\mathrm{C}_{15} \mathrm{H}_{26}$ & 1715.385 & 1.30 \\
\hline 48 & $\begin{array}{l}\text { 1-Ethenyl-1-methyl-2-(1-methylethenyl)-4-(1-methylethylidene)- } \\
\text { cyclohexane }\end{array}$ & 17.72 & $\mathrm{C}_{15} \mathrm{H}_{24}$ & 1725.000 & 0.82 \\
\hline 49 & $\alpha$-Farnesene & 17.94 & $\mathrm{C}_{15} \mathrm{H}_{24}$ & 1735.577 & 0.13 \\
\hline 50 & 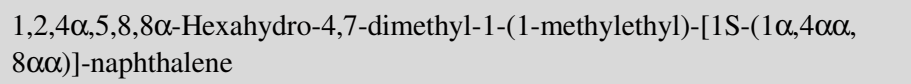 & 18.20 & $\mathrm{C}_{15} \mathrm{H}_{24}$ & 1748.077 & 2.20 \\
\hline 51 & 1-(1,5-Dimethyl-4-hexenyl)-4-methyl-benzene & 18.48 & $\mathrm{C}_{15} \mathrm{H}_{22}$ & 1761.538 & 0.19 \\
\hline 52 & 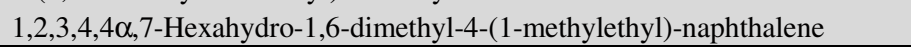 & 18.70 & $\mathrm{C}_{15} \mathrm{H}_{24}$ & 1772.115 & 0.20 \\
\hline
\end{tabular}




\begin{tabular}{|c|c|c|c|c|c|}
\hline 53 & 1,7,7-Trimethyl-(1S-endo)-bicyclo[2.2.1]heptan-2-ol & 19.00 & $\mathrm{C}_{10} \mathrm{H}_{18} \mathrm{O}$ & 1786.538 & 0.16 \\
\hline 54 & 1,2,3,4-Tetrahydro-1,6-dimethyl-4-(1-methylethyl)-, (1S-cis)-naphthalene & 19.71 & $\mathrm{C}_{15} \mathrm{H}_{22}$ & 1821.078 & 0.39 \\
\hline 55 & 2-Methyl-5-(1-methylethenyl)-trans-2-cyclohexen-1-ol & 19.83 & $\mathrm{C}_{10} \mathrm{H}_{16} \mathrm{O}$ & 1826.961 & 0.17 \\
\hline 56 & Benzocycloheptatriene & 20.06 & $\mathrm{C}_{11} \mathrm{H}_{10}$ & 1838.235 & 0.16 \\
\hline 57 & 1-Hydroxy-1,7-dimethyl-4-isopropyl-2,7-cyclodecadiene & 20.89 & $\mathrm{C}_{15} \mathrm{H}_{26} \mathrm{O}$ & 1878.922 & 0.74 \\
\hline 58 & Caryophyllene oxide & 22.75 & $\mathrm{C}_{15} \mathrm{H}_{24} \mathrm{O}$ & 1974.093 & 1.53 \\
\hline 64 & 1,2-Dimethoxy-4-(2-propenyl)-benzene & 23.23 & $\mathrm{C}_{11} \mathrm{H}_{14} \mathrm{O}_{2}$ & 1998.964 & 0.32 \\
\hline 59 & $\begin{array}{l}\text { 1,5,5,8-Tetramethyl-[1R-(1R*,3E,7E,11R*)]-12-oxabicyclo[9.1.0]dodeca- } \\
\text { 3,7-diene }\end{array}$ & 23.72 & $\mathrm{C}_{15} \mathrm{H}_{24} \mathrm{O}$ & 2029.936 & 0.69 \\
\hline 60 & 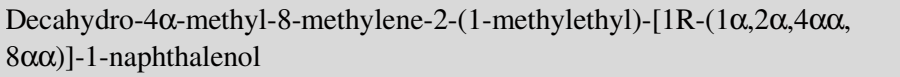 & 24.04 & $\mathrm{C}_{15} \mathrm{H}_{26} \mathrm{O}$ & 2050.318 & 0.62 \\
\hline 61 & $\begin{array}{l}\text { Decahydro-4,8,8-trimethyl-[1S-( } 1 \alpha, 3 \alpha \alpha, 4 \alpha, 8 \alpha \alpha, 9 \mathrm{R} *)]-1,4 \text {-methanoazulene } \\
\text {-9-methanol }\end{array}$ & 24.86 & $\mathrm{C}_{15} \mathrm{H}_{26} \mathrm{O}$ & 2103.774 & 0.17 \\
\hline 62 & $8 \alpha \mathrm{H}$-cedran-8-ol & 24.93 & $\mathrm{C}_{15} \mathrm{H}_{26} \mathrm{O}$ & 2110.377 & 0.61 \\
\hline 63 & (-)-Spathulenol & 25.02 & $\mathrm{C}_{15} \mathrm{H}_{24} \mathrm{O}$ & 2118.868 & 2.32 \\
\hline 65 & 3-(1-Methyl-1H-pyrrol-2-yl)-pyridine & 27.50 & $\mathrm{C}_{12} \mathrm{H}_{10} \mathrm{~N}_{2}$ & 2321.569 & 0.13 \\
\hline 66 & Caryophyllene oxide & 27.61 & $\mathrm{C}_{15} \mathrm{H}_{24} \mathrm{O}$ & 2332.353 & 0.17 \\
\hline 67 & Aromadendrene oxide-(2) & 28.02 & $\mathrm{C}_{15} \mathrm{H}_{24} \mathrm{O}$ & 2372.549 & 0.22 \\
\hline 68 & $\begin{array}{l}1,2,3,4,4 \alpha, 9,10,10 \alpha \text {-octahydro-1,4 } \alpha \text {-dimethyl-7-(1-methylethyl)-[1S- } \\
(1 \alpha, 4 \alpha \alpha, 10 \alpha \alpha)] \text {-1-phenanthrenemethanol }\end{array}$ & 29.13 & $\mathrm{C}_{20} \mathrm{H}_{30} \mathrm{O}$ & 2484.553 & 1.09 \\
\hline Total & & & & & 98.51 \\
\hline
\end{tabular}

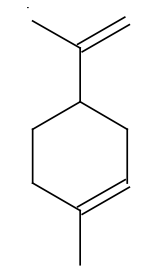

Component 1

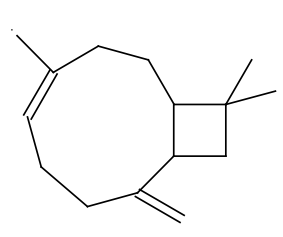

Component 2

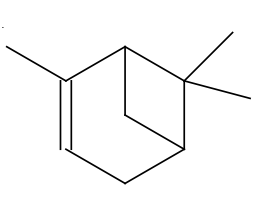

Component 3

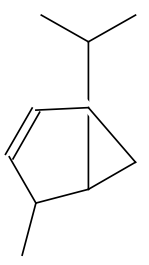

Component 4

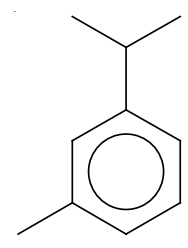

Component 5

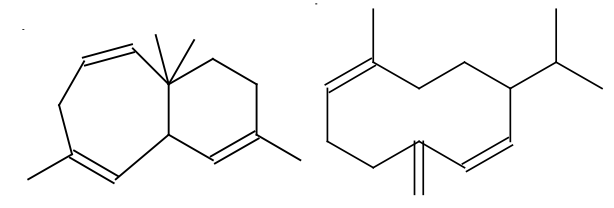

Component 6

Component 7<smiles>CC1=CCC2C3CCCC1C3C2C(C)C</smiles>

Component 8

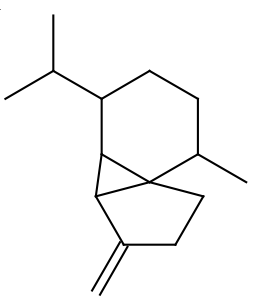

Component 9<smiles>CC1=CCC2C(C)=CCC(C(C)C)C2C1</smiles>

Component 10

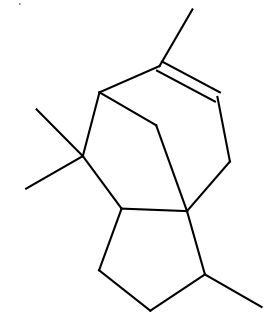

Component 11

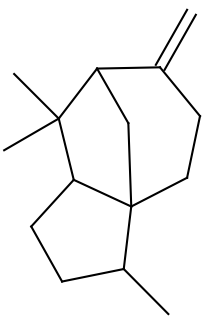

Component 12

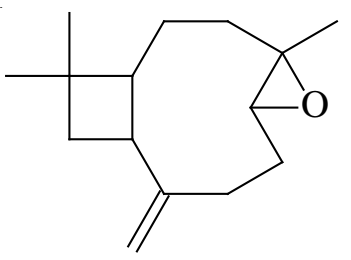

Component 13

Fig. 2. Representative volatile components of essential oils from Wedelia biflora

The determined 68 chemical components are retrieved through the computer bank. The similarities of 65 kinds of chemical composition are over $80 \%$ (except that only 3 chemical components have the similarity of nearly $75 \%$ ), which shows that it has the relatively high reliability.

The main chemical components of the volatile oil in the Wedelia biflora (Linn.) DC. by the method of SPME-GC/MS are the terpenoids and the micro-molecular aromatic compounds like single terpene and diterpene, etc. Most of these diterpene compounds have strong fragrance and various bioactivities. For example, D-limonene has the strong citrus smell and the insecticidal action, which can be used as aromatic additives besides being used as botanical insecticides. All in all, through the method of solid-phase microextraction, SPME and the gas chromatograph-tandem mass spectrometer, it almost completely reflects the chemical components of the volatile oil in the Wedelia biflora (Linn.) DC. and thus it provides the reference data for its further development and utilization.

\section{ACKNOWLEDGEMENTS}

The authors are grateful to the Center of Analysis and Testing, Jiangnan University, for performing GC-MS.

\section{REFERENCES}

1. Y.Q. Wu, Y.J. Hu and F.L. Liao, Guihaia, 25, 5 (2005).

2. G.Y. Li, X.W. Xian, X. Deng, Z. Wang, G.H. Zhang and Q.F. Li, Chin. Agric. Sci. Bull., 26, 1 (2010).

3. D.J. Yang, S.J. Guo, Y.P. Chen and W.B. Zhang, J. Northwest Forestry Univ., 25, 1 (2010).

4. M.Y. Liu, J.Y. Zhu and X.B. Jin, China Pharm., 22, 11 (2011).

5. T. Zhang, G.H. Du and R.Y. Chen, Chin. Pharm. J., 45, 24 (2010).

6. F. Yu, S.M. Wang, M. Dong, Z.Y. Ni, F.J. Dong, Q.W. Shi, X.F. Lingmu and B. Cong, Nat. Prod. Res. Dev., 22 (2010).

7. S.X. Jiang and J.J. Feng, Chin. J. Chromatogr., 30, 3 (2012).

8. J.L. Hu, P. Zheng and L. Hua, J. Hubei Univ. Educ., 29, 2 (2012).

9. W.C. Xie, X.H. Yang, C.H. Zhang, H.W. Ji and L.F. Zhang, Chin. J. Anal. Chem., 39, 12 (2010).

10. Z. He, Z.M. Lu, H.Y. Xu, J.S. Shi and Z.H. Xu, J. Chin. Med. Mater., 34, 11 (2011) 